\title{
Hyperaldosteronism: types, clinical characteristics, and treatment
}

\author{
Areej M. Alsyamy ${ }^{1 *}$, Amnah T. Kashkari ${ }^{2}$, Fawaz A. Alazdi $^{3}$, Abdulmonem T. Dalati ${ }^{4}$, \\ Mohammed M. Alawadi ${ }^{4}$, Emad S. Hawari ${ }^{4}$, Mohammed M. Nassar ${ }^{4}$, \\ M. Mohanad I. A. Hennawi ${ }^{4}$, Hassan H. Alaslani ${ }^{5}$, Ashjan A. Albayshi ${ }^{6}$, Mohammed L. Fatani ${ }^{7}$
}

\author{
${ }^{1}$ Department of Internal Medicine, King Abdullah Medical Complex, Jeddah, Saudi Arabia \\ ${ }^{2}$ College of Medicine, Taibah University, Medina, Saudi Arabia \\ ${ }^{3}$ College of Medicine, King Abdulaziz University, Jeddah, Saudi Arabia \\ ${ }^{4}$ College of Medicine, Alfaisal University, Riyadh, Saudi Arabia \\ ${ }^{5}$ Department of Internal Medicine, Eradah Mental Health Complex, Jeddah, Saudi Arabia \\ ${ }^{6}$ College of Medicine, Imam Mohammad Ibn Saud Islamic University, Riyadh, Saudi Arabia \\ ${ }^{7}$ Department of Internal Medicine, King Abdulaziz Hospital, Jeddah, Saudi Arabia
}

Received: 15 November 2021

Accepted: 29 November 2021

\section{*Correspondence:}

Dr. Areej M. Alsyamy,

E-mail: areej.msss@hotmail.com

Copyright: (C) the author(s), publisher and licensee Medip Academy. This is an open-access article distributed under the terms of the Creative Commons Attribution Non-Commercial License, which permits unrestricted non-commercial use, distribution, and reproduction in any medium, provided the original work is properly cited.

\begin{abstract}
Cases with hyperaldosteronism might be asymptomatic in many events. Therefore, the diagnosis can be missed. However, the usual presentation for many patients has been reported to be a refractory elevation in the blood pressure which might be mild to severe. Based on the type of hyperaldosteronism and the diagnosis, the treatment of these conditions should be established. Therefore, it can be concluded that the treatment is specific to the management of the underlying etiology, and managing the clinical characteristics and associated complications. This present literature review aims to provide evidence regarding the types, clinical characteristics, and treatment of aldosterone based on data from the current investigations in the literature. Different clinical phenotypes have been reported for the condition. Nevertheless, the disease can be broadly classified into primary and secondary hyperaldosteronism based on the pathophysiology and etiology of the condition. Clinical characteristics might not be diagnostic since they are very nonspecific, despite being common in these patients, as hypokalemia and hypertension. Therefore, clinicians should be aware of conducting the necessary diagnostic approaches before establishing the diagnosis. Management of these patients requires the integration of different approaches, including surgical and medical treatment. Perioperative care is important because it may lead to unfavorable consequences if neglected.
\end{abstract}

Keywords: Hyperaldosteronism, Conn syndrome, Mineralocorticoids, Adrenal hyperplasia, Renal artery stenosis, Aldosterone, Treatment, Clinical presentation

\section{INTRODUCTION}

Aldosterone is a hormone secreted from the outer layer of the adrenal cortex (zone glomerulosa) as the most significant mineralocorticoid. Hyperaldosteronism develops when increased secretion of the hormone occurs within the body above the normal levels. Cases with hyperaldosteronism might be asymptomatic in many events. Consequently, the diagnosis can be missed.
Nevertheless, the usual presentation for many patients has been reported to be a refractory elevation in the blood pressure, which might be mild to severe. ${ }^{1}$

There are two main types of hyperaldosteronism, which usually have similar clinical characteristics and are difficult to be clinically differentiated. The diagnosis of these conditions is mainly based on laboratory and imaging diagnostic evaluations. ${ }^{2}$ However, it should be noted that 
the underlying etiology is different, and many causes were proposed for each type. Based on the type of hyperaldosteronism and the diagnosis, the treatment of these conditions should be established. Therefore, it can be concluded that the treatment is specific to the management of the underlying etiology, and managing the clinical characteristics and associated complications. ${ }^{3-6}$ This present literature review aims to provide evidence regarding the types, clinical characteristics, and treatment of aldosterone based on data from the current investigations in the literature.

\section{METHODS}

This literature review is based on an extensive literature search in Medline, Cochrane, and EMBASE databases which was performed on 27 October 2021 using the medical subject headings (MeSH) or a combination of all possible related terms, according to the database. To avoid missing potential studies, a further manual search for papers was done through Google Scholar while the reference lists of the initially included papers. Papers discussing hyperaldosteronism types, clinical characteristics, and treatment were screened for useful information. No limitations were posed on date, language, age of participants, or publication type.

\section{DISCUSSION}

\section{Types}

The clinical presentation of hyperaldosteronism vary hugely based on the type of the underlying pathology and the prognosis of the cases. Two main types of hyperaldosteronism were reported in the literature and are clinically discovered. These include primary and secondary hyperaldosteronism. ${ }^{7}$ Increased production of aldosterone from the adrenal gland, particularly zona glomerulosa, has been associated with the development and pathology of primary hyperaldosteronism. The etiology of the condition can be either attributed to the presence of an adrenal tumor in the adrenal gland, which has been called Conn syndrome, or as an adrenal bilateral hyperplasia. $^{8,9}$ Familial hyperaldosteronism type 1, aldosterone-producing adrenocortical carcinomas, ectopic aldosterone-secreting tumors, and unilateral adrenal hyperplasia are other forms of primary hyperaldosteronism. However, estimates show that these are not very common and are rarely seen in clinical settings. Evidence indicates that idiopathic bilateral adrenal hyperplasia is the commonest cause of increased aldosterone production by the adrenal glands leading to the development of primary hyperaldosteronism (as estimated in around $2 / 3$ of the cases with primary hyperaldosteronism). ${ }^{10,11}$ Other cases might be attributed to the presence of Conn syndrome, while other reported etiologies are rare. Patients with primary hyperaldosteronism are usually asymptomatic. Nonetheless, reports show that hypertension and hypokalemia might be the main clinical manifestations in these patients. Estimates show that the prevalence of primary hyperaldosteronism is variable across the different populations, and the rate ranges between $4.6 \%$ and $16.6 \%$. These differences are attributed to the severity of hypertension and underlying clinical manifestations, diagnostic methods, and patient selection. ${ }^{12}$ It has been furtherly demonstrated that among patients with hypertension, the prevalence of primary hyperaldosteronism was found to be $10 \% \cdot{ }^{13}$ Still, evidence also shows that the rate is different and further studies are needed for verification.

On the other hand, excess activation of the reninangiotensin-aldosterone system (RAAS) leads to the development and initiation of the pathology of secondary hyperaldosteronism (Figure 1). This activation has been reported secondary to many causes, including renal artery stenosis, renin-producing tumors, or edematous disorders, including cirrhosis with ascites, cor pulmonale, pregnancy, and left ventricular heart failure. The excess activation of the RAAS may be transient (secondary to temporary pathologies as hypovolemia) or sustained (secondary to permanent pathologies that lead to sustained activation of the RAAS). Fibromuscular dysplasia or atherosclerosisinduced renal artery stenosis can significantly initiate a state of false hypovolemia due to reduced blood flow to the kidneys, which will cumulatively lead to increased aldosterone secretion. ${ }^{14}$ Stimulating aldosterone secretion was also reported secondary to cases when cardiac output is reduced, which occurs in cases of cor pulmonale or leftsided (congestive) heart failure. Reduced perfusion and blood flow through the kidneys might also develop secondary to the presence of cirrhosis and ascites, leading to a remarkable reduction in the volume of the circulating fluid, which will also impact the amount of aldosterone secretion. A renin-producing tumor was also reported to be a potential cause for the development of secondary hyperaldosteronism and is usually detected in juxtaglomerular cells, but estimates show that the prevalence of the condition is relatively rare. ${ }^{15}$ Epidemiological studies indicate that the prevalence of secondary hyperaldosteronism is remarkably lower than the prevalence of the primary form. Furthermore, evidence indicates that women are more frequently affected with both primary and secondary hyperaldosteronism. ${ }^{13,16}$

\section{Clinical characteristics}

Both primary and secondary hyperaldosteronism might present with hugely variable clinical manifestations. Hypertension might be the main clinical manifestation among patients with hyperaldosteronism. Hypokalemia might also be present due to excessive renal potassium wasting. The presence of comorbidities, the amount of aldosterone secretion, and the underlying cause are different factors that can significantly determine the clinical presentation and phenotypes of hyperaldosteronism. In addition to hypertension and 
hypokalemia, patients with hyperaldosteronism usually suffer from metabolic alkalosis, as well. This is usually found among patients with primary hyperaldosteronism. Hypertension is very common among patients with primary hyperaldosteronism and usually results from increased peripheral vascular resistance, and plasma volume expansion secondary to the excess aldosterone secretion. In many cases, hypertension might be severe, and evidence shows that some patients might suffer from a refractory condition. ${ }^{17}$ However, hypertension might not be present in many cases, and some patients only suffer from slight elevations in the blood pressure. Therefore, the diagnosis of hyperaldosteronism should not be limited to the presence of hypertension. ${ }^{18-23}$ The spontaneous development of hypokalemia is a strong indicator for the presence of hyperaldosteronism, irrespective of the presence of hypertension in the affected patient. Following the administration of potassium-wasting diuretics (including furosemide or hydrochlorothiazide), patients should be investigated and managed for severe hypokalemia. However, previous studies also reported that most cases with primary hyperaldosteronism usually have normal potassium levels. ${ }^{17,24}$

Hyperaldosteronism also results in increased sodium reabsorption, leading to secondary extracellular expansion. Still, at a certain level, the kidneys begin to excrete excess sodium which encounters the action of excess aldosterone due to the increased atrial natriuretic peptide secretion. Accordingly, among patients with normal renal and cardiac functions, peripheral edema is usually present in cases of primary hyperaldosteronism. Besides, metabolic alkalosis usually manifests in these patients due to the increased secretion of urinary hydrogen ions from the renal distal tubules. It should be noted that the condition is not usually noticed, being mild in most cases, and does not have any clinical significance. ${ }^{10,20}$ Mild hypernatremia and hypomagnesemia might also be present in these patients, which has been attributed to the potential reset of the osmostat. Neuromuscular symptoms as weakness and paraesthesias are also reported for patients with hyperaldosteronism. However, estimates indicate that these manifestations are not common in this context. These manifestations are attributed to the significant disturbances in the electrolyte levels causes by hyperaldosteronism. Resistance to the renal tubular antidiuretic hormone in the renal tubules (secondary to the presence of hypokalemia) might also lead to the development of nephrogenic diabetes insipidus. This has been associated with clinical manifestations, including polydipsia, mild polyuria, and nocturia. Cardiac arrhythmias and atrial fibrillation might also manifest among patients with hyperaldosteronism and can even lead to death. However, the frequency of these complications is rare in clinical settings. ${ }^{13}$

\section{Treatment}

Treating patients with hyperaldosteronism is mainly based on the underlying etiology. The aim of conducting the treatment approaches is to manage the serious underlying complications that might have resulted from chronic aldosterone secretion. For instance, the management of cardiovascular events can be life-saving in these situations, and in such cases management of the different cardiovascular events as atrial fibrillation, heart failure, myocardial infarction, stroke, and left ventricular hypertrophy is critical to enhancing the prognosis. Besides, blood pressure and serum potassium levels should be normalized, which were noticed to potentially persist even after correction of hyperaldosteronism. In most cases, surgery is the treatment of choice for aldosteroneproducing adenoma, which is usually done by either anterior or posterior laparoscopic approaches. ${ }^{25,26}$ The surgery has been reported to reduce the time of hospital stay and enhance patient recovery. In unilateral cases, it has been demonstrated that radiofrequency ablation has been reported as an efficacious alternative to the surgical approach, which has been reported with and effective longterm outcomes, including the normalization of the potassium and blood pressure levels, which was reported to be similar to the outcomes obtained with surgical resection. Furthermore, it has been shown that this approach can significantly reduce the length of hospital stay. ${ }^{27,28}$ On the other hand, other studies reported that many adverse events were reported with this approach, including adrenal abscess formation, pneumothorax, pancreatitis, hematuria, abdominal pain, and hypertensive episodes. ${ }^{29,30}$ Although favorable outcomes were reported with the surgical and laparoscopic modalities, pre-and post-operative caution should be considered, and palliative care should be adequately conducted for the affected patients with aldosterone-producing adenoma. ${ }^{31}$ For instance, mineralocorticoid-receptor antagonists can be added in the preoperative setting for adequate control of hypokalemia and hypertension. A transient hyporeninemic hypoaldosteronism state can develop postoperatively due to the reduced aldosterone secretion in the corresponding adrenal gland. Therefore, antagonists for potassium and mineralocorticoid receptors should also be administered postoperatively. The expected clinical manifestations postoperatively in these events include orthostatic hypotension, mild dehydration, mild hyperkalemia, and salt-wasting. ${ }^{32}$ The management of bilateral adrenal hyperplasia has been reported to be medically approached by the administration of mineralocorticoid receptor antagonists. Conversely, it should be noted that surgical resection might be considered in cases of asymmetric adrenal hyperplasia. However, perioperative care should be offered to these patients as previously discussed to maintain enhanced overall outcomes. ${ }^{33,34}$

It should be noted that although the administration of mineralocorticoid-receptor antagonists has been reported with favorable outcomes, evidence indicates that these modalities are usually used with caution because of the potential to develop several adverse events. For instance, erectile dysfunction, and gynecomastia were reported among male patients receiving spironolactone for a long 
period, while primarily intermenstrual bleeding, menstrual dysfunction, gastrointestinal symptoms, and fatigue were reported among females receiving the drug. ${ }^{31,35,36}$ Accordingly, some reports suggested that eplerenone should be administered instead due to the reduced risk to develop the reported adverse events as spironolactone. ${ }^{37,38}$ It has been furtherly demonstrated that the administration of high doses of mineralocorticoid receptor antagonists has been associated with more favorable outcomes, including a significant reduction in the risk of developing cardiovascular events. ${ }^{39-41}$

Conservative treatment should also be conducted for the management of hypokalemia and hypertension. Furthermore, the administration of glucocorticoids can be approached in cases of glucocorticoid-remediable aldosteronism. ${ }^{42}$ In cases with secondary hyperaldosteronism, management of the cause is also important to enhance the outcomes. For instance, medical treatment can be used to manage renal artery stenosis, either alone or in combination with revascularization. ${ }^{43,44}$
Primary angioplasty can also be used as the primary management modality for cases with renal artery fibromuscular dysplasia. Angioplasty with stent placement can also be offered for patients with atherosclerotic diseases. On the other hand, a clinical trial reported that revascularization was associated with better outcomes regarding enhanced survival rates for these patients. It should also be noted that medical therapy can be sufficient in cases with atherosclerotic renovascular hypertension. ${ }^{45}$

Finally, the administration of angiotensin receptor blockers and angiotensin-converting enzymes should be considered to normalize the levels of renin and reduce its activities. In addition to reducing the effects of renin, these modalities can also normalize the blood pressure levels, and produce various reno-protective effects.

However, they should be administered with caution to avoid the development of any complications that might deteriorate the prognosis. ${ }^{16,43}$

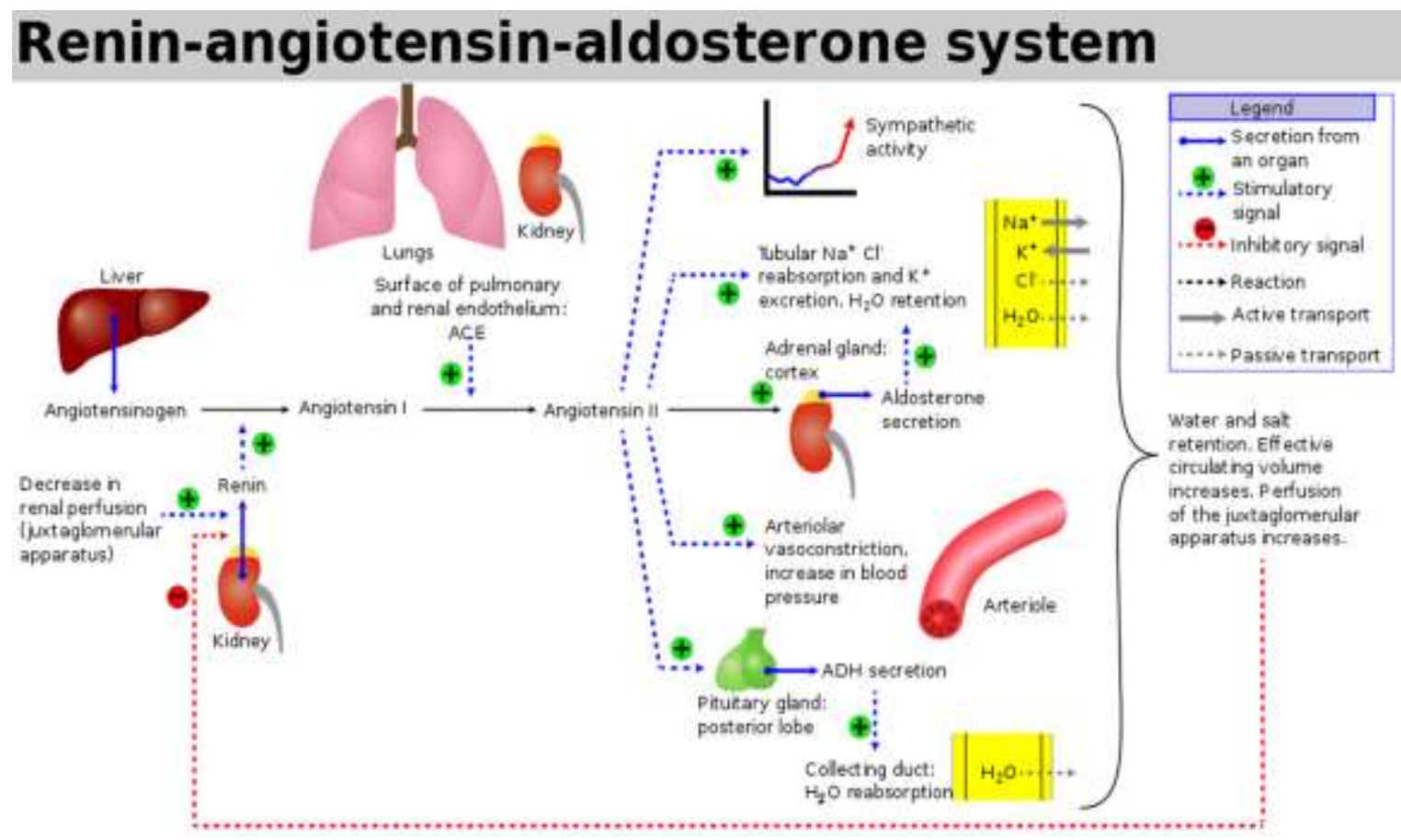

Figure 1. Renin-angiotensin-aldosterone system (RAAS).

\section{CONCLUSION}

Clinical characteristics of hyperaldosteronism might not be diagnostic since they are very non-specific, despite being common in these patients, as hypokalemia and hypertension. Therefore, clinicians should be aware of conducting the necessary diagnostic approaches before establishing the diagnosis. Management of these patients requires the integration of different approaches, including surgical and medical treatment. Perioperative care is important because it may lead to unfavorable consequences if neglected.
Funding: No funding sources

Conflict of interest: None declared

Ethical approval: Not required

\section{REFERENCES}

1. Scholl UI. Hyperaldosteronismus. Der Internist. 2021;62(3):245-51.

2. Vilela LAP, Almeida MQ. Diagnosis and management of primary aldosteronism. Arch Endocrinol Metabol. 2017;61(3):305-12. 
3. Chikladze NM, Favorova OO, Chazova IE. Family hyperaldosteronism type I: a clinical case and review of literature. Terapevticheskii arkhiv. 2018;90(9):115-22.

4. Stowasser M, Wolley M, Wu A, et al. Pathogenesis of Familial Hyperaldosteronism Type II: New Concepts Involving Anion Channels. Curr Hypertens Rep. 2019;21(4):31.

5. Pons Fernández N, Moreno F, Morata J. Familial hyperaldosteronism type III a novel case and review of literature. Rev Endocrine Metabol Disord. 2019;20(1):27-36.

6. Corssmit EPM, Dekkers OM. Screening in adrenal tumors. Curr Opinion Oncol. 2019;31(3):243-6.

7. Morera J, Reznik Y. Management Of Endocrine Disease: The role of confirmatory tests in the diagnosis of primary aldosteronism. Eur $\mathrm{J}$ Endocrinol. 2019;180(2):45-58.

8. Nishimoto $K$, Nakagawa $K$, Li $D$, et al. Adrenocortical zonation in humans under normal and pathological conditions. J Clin Endocrinol Metabol. 2010;95(5):2296-305.

9. Nanba K, Vaidya A, Williams GH, Zheng I, Else T, Rainey WE. Age-Related Autonomous Aldosteronism. Circulation. 2017;136(4):347-55.

10. Meyer A, Brabant G, Behrend M. Long-term followup after adrenalectomy for primary aldosteronism. World J Surg. 2005;29(2):155-9.

11. Streeten DH, Tomycz N, Anderson GH. Reliability of screening methods for the diagnosis of primary aldosteronism. Am J Med. 1979;67(3):403-13.

12. Prejbisz A, Warchoł-Celińska E, Lenders JW, Januszewicz A. Cardiovascular Risk in Primary Hyperaldosteronism. Hormone Metabol Res. 2015;47(13):973-80.

13. Papadopoulou-Marketou N, Vaidya A, Dluhy R, Chrousos GP. Hyperaldosteronism. In: Feingold KR, Anawalt B, Boyce A, editors. Endotext. South Dartmouth (MA): MDText.com, Inc. 2000.

14. Safian RD, Textor SC. Renal-artery stenosis. N Engl J Med. 2001;344(6):431-42.

15. Conn JW, Cohen EL, Lucas CP. Primary reninism. Hypertension, hyperreninemia, and secondary aldosteronism due to renin-producing juxtaglomerular cell tumors. Arch Internal Med. 1972;130(5):682-96.

16. Safian RD. Atherosclerotic Renal Artery Stenosis. Curr Treatment Options Cardiovasc Med. 2003;5(2):91-101.

17. Funder JW, Carey RM, Fardella C. Case detection, diagnosis, and treatment of patients with primary aldosteronism: an endocrine society clinical practice guideline. J Clin Endocrinol Metabol. 2008;93(9):3266-81.

18. Vaidya A, Underwood PC, Hopkins PN. Abnormal aldosterone physiology and cardiometabolic risk factors. Hypertension. 2013;61(4):886-93.

19. Baudrand R, Guarda FJ, Torrey J, Williams G, Vaidya A. Dietary Sodium Restriction Increases the Risk of Misinterpreting Mild Cases of Primary
Aldosteronism. J Clin Endocrinol Metabol. 2016;101(11):3989-96.

20. Markou A, Pappa T, Kaltsas G. Evidence of primary aldosteronism in a predominantly female cohort of normotensive individuals: a very high odds ratio for progression into arterial hypertension. J Clin Endocrinol Metabol. 2013;98(4):1409-16.

21. Brown JM, Underwood PC, Ferri C. Aldosterone dysregulation with aging predicts renal vascular function and cardiovascular risk. Hypertension. 2014;63(6):1205-11.

22. Kono T, Ikeda F, Oseko F, Imura H, Tanimura H. Normotensive primary aldosteronism: report of a case. J Clin Endocrinol Metabol. 1981;52(5):1009-13.

23. El-Qushayri AE, Ghozy S, Reda A, Kamel AMA, Abbas AS, Dmytriw AA. The impact of Parkinson's disease on manifestations and outcomes of Covid-19 patients: A systematic review and meta-analysis. Rev Med Virol. 2021;2278.

24. Mulatero P, Stowasser M, Loh KC. Increased diagnosis of primary aldosteronism, including surgically correctable forms, in centers from five continents. J Clin Endocrinol Metabol. 2004;89(3):1045-50.

25. Nehs MA, Ruan DT. Minimally invasive adrenal surgery: an update. Curr Opinion Endocrinol Diabetes Obesity. 2011;18(3):193-7.

26. Thieu H, Bach Dat B, Nam NH. Antibiotic resistance of Helicobacter pylori infection in a children's hospital in Vietnam: prevalence and associated factors. Minerva Medica. 2020;111(5):498-501.

27. Liu SY, Chu CC, Tsui TK. Aldosterone-producing Adenoma in Primary Aldosteronism: CT-guided Radiofrequency Ablation-Long-term Results and Recurrence Rate. Radiology. 2016;281(2):625-34.

28. Sarwar A, Brook OR, Vaidya A. Clinical Outcomes following Percutaneous Radiofrequency Ablation of Unilateral Aldosterone-Producing Adenoma: Comparison with Adrenalectomy. J Vasc Intervent Radiol. 2016;27(7):961-7.

29. Yang MH, Tyan YS, Huang YH, Wang SC, Chen SL. Comparison of radiofrequency ablation versus laparoscopic adrenalectomy for benign aldosteroneproducing adenoma. La Radiologia medica. 2016;121(10):811-9.

30. Keeling AN, Sabharwal T, Allen MJ, Hegarty NJ, Adam A. Hypertensive crisis during radiofrequency ablation of the adrenal gland. J Vasc Intervent Radiol. 2009;20(7):990-1.

31. Funder JW, Carey RM, Mantero F. The Management of Primary Aldosteronism: Case Detection, Diagnosis, and Treatment: An Endocrine Society Clinical Practice Guideline. J Clin Endocrinol Metabol. 2016;101(5):1889-916.

32. Lim PO, Young WF, MacDonald TM. A review of the medical treatment of primary aldosteronism. J Hypertens. 2001;19(3):353-61. 
33. Crane MG, Harris JJ. Effect of spironolactone in hypertensive patients. Am J Med Sci. 1970;260(6):311-30.

34. Brown JJ, Davies DL, Ferriss JB. Comparison of surgery and prolonged spironolactone therapy in patients with hypertension, aldosterone excess, and low plasma renin. Br Med J. 1972;2(5816):729-34.

35. Karagiannis A, Tziomalos K, Papageorgiou A. Spironolactone versus eplerenone for the treatment of idiopathic hyperaldosteronism. Expert Opinion Pharmacotherapy. 2008;9(4):509-15.

36. Jeunemaitre X, Chatellier G, Kreft-Jais C. Efficacy and tolerance of spironolactone in essential hypertension. Am J Cardiol. 1987;60(10):820-5.

37. Tam TS, Wu MH, Masson SC. Eplerenone for hypertension. Cochrane Database Systemat Rev. 2017;2(2):Cd008996.

38. Parthasarathy HK, Ménard J, White WB. A doubleblind, randomized study comparing the antihypertensive effect of eplerenone and spironolactone in patients with hypertension and evidence of primary aldosteronism. J Hypertens. 2011;29(5):980-90.

39. Hundemer GL, Vaidya A. Primary Aldosteronism Diagnosis and Management: A Clinical Approach. Endocrinol Metabolism Clinics North Am. 2019;48(4):681-700.

40. Hundemer GL. Primary Aldosteronism: Cardiovascular Outcomes Pre- and Post-treatment. Curr Cardiol Rep. 2019;21(9):93.

41. Son PT, Reda A, Viet DC. Exchange transfusion in the management of critical pertussis in young infants: a case series. Vox Sang. 2021.
42. Dluhy RG, Lifton RP. Glucocorticoid-remediable aldosteronism. J Clin Endocrinol Metabol. 1999;84(12):4341-4.

43. Hirsch AT, Haskal ZJ, Hertzer NR. ACC/AHA 2005 Practice Guidelines for the management of patients with peripheral arterial disease (lower extremity, renal, mesenteric, and abdominal aortic): a collaborative report from the American Association for Vascular Surgery/Society for Vascular Surgery, Society for Cardiovascular Angiography and Interventions, Society for Vascular Medicine and Biology, Society of Interventional Radiology, and the ACC/AHA Task Force on Practice Guidelines (Writing Committee to Develop Guidelines for the Management of Patients With Peripheral Arterial Disease): endorsed by the American Association of Cardiovascular and Pulmonary Rehabilitation; National Heart, Lung, and Blood Institute; Society for Vascular Nursing; TransAtlantic Inter-Society Consensus; and Vascular Disease Foundation. Circulation. 2006;113(11):463-654.

44. Nally JV, Olin JW, Lammert GK. Advances in noninvasive screening for renovascular disease. Cleveland Clin J Med. 1994;61(5):328-36.

45. Edwards MS, Corriere MA. Contemporary management of atherosclerotic renovascular disease. J Vasc Surg. 2009;50(5):1197-210.

Cite this article as: Alsyamy AM, Kashkari AT, Alazdi FA, Dalati AT, Alawadi MM, Hawari ES, et al. Hyperaldosteronism: types, clinical characteristics, and treatment. Int J Community Med Public Health 2022;9:420-5. 